Pacific Journal of Mathematics

ON THE ABSOLUTE BAIRE PROPERTY 


\title{
ON THE ABSOLUTE BAIRE PROPERTY
}

\author{
JoHN C. MORGAN II
}

\begin{abstract}
In earlier papers the author has formulated an axiomatic foundation for an abstract theory of Baire category, one of whose purposes is the unification of analogous concepts and theorems occurring in topology and measure theory. In the present article, a method is presented for unifying, within this axiomatic framework, the Baire property in the restricted sense and absolute measurability in complete separable metric spaces.
\end{abstract}

In [14] we have defined the absolute Baire property in order to unify the concepts of the Baire property in the restricted sense and absolute measurability on the real line. After recalling this general definition in $\S 2$, we show how these two concepts can also be unified for linear sets using a special case of the general definition which involves order isomorphic mappings of the set of all irrational numbers into the real line and which we call the ordinal version of the absolute Baire property. In $\S 3$ we formulate the appropriate topological analogue, utilizing homeomorphism of the set of irrational numbers, of this ordinal version and show this topological version of the absolute Baire property serves to unify these two concepts in the setting of complete separable metric spaces. A general theorem on the topological invariance in complete metric spaces of this topological version is then proved in $\S 4$.

1. Preliminaries. For the convenience of the reader, we include in this section pertinent notation, terminology, and results contained in [13] and [14].

Notation. If $\mathscr{S}$ is any family of sets, then the elements of $\mathscr{S}$ will be called $\mathscr{S}$-sets.

Definition 1. A family $\mathscr{C}$ of subsets of a nonempty set $X$ is called a $\Re$-family if the following axioms are satisfied.

1. $X=\bigcup \mathscr{C}$.

2. Let $A$ be a $\mathscr{C}$-set and let $\mathscr{D}$ be a nonempty family of disjoint $\mathscr{C}$-sets which has power less than the power of $\mathscr{C}$.

(a) If $A \cap(\bigcup \mathscr{D})$ contains a $\mathscr{C}$-set, then there is a $\mathscr{D}$-set $D$ such that $A \cap D$ contains a $\mathscr{C}$-set.

(b) If $A \cap(\bigcup \mathscr{D})$ contains no $\mathscr{C}$-set, then there is a $\mathscr{C}$-set $B \subset A$ which is disjoint from all $\mathscr{D}$-sets. 
The symbol $\mathscr{C}$ will always signify a $\Omega$-family with respect to which we define generalized Baire category concepts in the following manner.

Definition 2. A set $S \subset X$ is $\mathscr{C}$-singular if each $\mathscr{C}$-set $A$ contains a $\mathscr{C}$-set $B$ which is disjoint from $S$. We denote by $\mathscr{C}_{I}$ the family of all countable unions of $\mathscr{C}$-singular sets and by $\mathscr{C}_{I I}$ the family of all subsets of $X$ which are not $\mathscr{C}_{I}$-sets.

A set $S \subset X$ is a $\mathscr{C}_{I I}$-set everywhere on a $\mathscr{C}$-set $A$ if $S \cap B$ is a $\mathscr{C}_{I I}$-set for every $\mathscr{C}$-set $B \subset A$. The set $S$ is a $\mathscr{C}_{I I}$-set everywhere if $S \cap B$ is a $\mathscr{C}_{I I}$-set for every $\mathscr{C}$-set $B$.

A set $S \subset X$ has the Baire property with respect to $\mathscr{C}$ if for every $\mathscr{C}$-set $A$ there is a $\mathscr{C}$-set $B \subset A$ such that either $B \cap S$ or $B \cap(X-S)$ is a $\mathscr{C}_{I}$-set.

The family of all subsets of $X$ which have the Baire property with respect to $\mathscr{C}$ will be denoted by $\mathfrak{B}(\mathscr{C})$. This family is a $\sigma$ field containing the family $\mathscr{C}$ and the $\sigma$-ideal of $\mathscr{C}_{I}$-sets.

We next recall three of the main examples of $\Omega$-families.

EXAMPLE 1. Let $\mathscr{C}$ be the family of all nonempty open sets in a topological space $X$. Then the $\mathscr{C}$-singular, $\mathscr{C}_{I}$-sets, and $\mathscr{C}_{I I}$-sets coincide with the sets which are nowhere dense, of the first Baire category, and of the second Baire category, respectively. The family $\mathfrak{B}(\mathscr{C})$ coincides with the family of all sets which have the classical topological Baire property (in the wide sense).

ExAMPle 2. Let $(X, \mathscr{A}, \mu)$ be a $\sigma$-finite measure space, let $\mathscr{C}$ be the family of all $\mu$-measurable sets of positive measure, and let $(X, \mathscr{A}, \bar{\mu})$ be the completion of $(X, \mathscr{A}, \mu)$. The classes of $\mathscr{C}$-singular and $\mathscr{C}_{1}$-sets are identical and coincide with the sets of $\bar{\mu}$-measure zero, the $\mathscr{C}_{I I}$-sets coincide with the subsets of $X$ which have positive outer $\bar{\mu}$-measure, and $\mathfrak{B}(\mathscr{C})$ coincides with the family $\bar{A}$ of all $\bar{\mu}$-measurable sets.

EXAMPLE 3. Let $X$ be an uncountable, complete separable metric space and let $\mathscr{C}$ be the family of all uncountable Borel sets. The $\mathscr{C}$-singular and $\mathscr{C}_{I}$-sets are identical and coincide with the sets having Marczewski's property $\left(s^{\circ}\right) . \quad \mathfrak{B}(\mathscr{C})$ coincides with the sets having Marczewski's property $(s)$ (see [24]).

In general, the families $\mathscr{C}_{I}, \mathscr{C}_{I I}$, and $\mathfrak{B}(\mathscr{C})$ are not uniquely determined by $\mathscr{C}$. Hence we are led to formulate the following notion of equivalent $\Re$-families.

Definition 3. Two $\Re$-families $\mathscr{C}$ and $\mathscr{D}$ of subsets of the same 
set $X$ are called equivalent if $\mathscr{C}_{I}=\mathscr{D}_{I}$ and $\mathfrak{B}(\mathscr{C})=\mathfrak{B}(\mathscr{D})$.

Examples of $\Re$-families which are equivalent to those of Examples 1-3 above, when $X$ is the real line with the usual topology and $\mu$ is the Borel-Lebesgue measure, are given in Examples $1 \mathrm{~A}-3 \mathrm{~A}$ in $\S 2$.

Finally we recall the most important theorem of our general theory of Baire category, which is a generalization of the so-called Banach Category Theorem (see [13] Theorem 2).

Fundamental Theorem. If $S$ is a $\mathscr{C}_{I I}$-set, then $S$ is a $\mathscr{C}_{I I}$-set everywhere on some $\mathscr{C}$-set.

2. The absolute Baire property: ordinal version. We first recall the definition of absolute $\mathscr{C}_{I}$-sets and the absolute Baire property given in [14] for linear sets. We shall use the letter $Z$, throughout this article, to denote the set of all irrational numbers, and the letter $X$ will, in this section, denote the set of all real numbers.

Definition 4. Let $(K, \mathscr{C})$ be a $\Re$-family, where $K$ is a subset of $X$. A set $S \subset X$ has the absolute Baire property with respect to $(K, \mathscr{C})$ [respectively, is an absolute $\mathscr{C}_{I}$-set] if for every order isomorphism $\phi$ mapping $K$ into $X$, the set $S \cap \phi(K)$ has the Baire property with respect to $(\phi(K), \phi(\mathscr{C}))$ [respectively, is a $\phi(\mathscr{C})_{I}$-set].

We shall denote the family of sets which have the absolute Baire property with respect to $(K, \mathscr{C})$ by $\mathfrak{A}(\mathscr{C})$.

The most important $\Re$-families $(K, \mathscr{C})$ are those for which $K=X$ and $\mathscr{C}$ consists of perfect sets satisfying the condition

(+) for every $\mathscr{C}$-set $A$ and every point $x \in A$, there is a descending sequence $\left\langle A_{n}\right\rangle_{n=1}^{\infty}$ of $\mathscr{C}$-sets such that $x \in A_{n}$, $A_{n} \subset A$, and $\operatorname{diam}\left(A_{n}\right) \leqq 1 / n$ for each $n$.

These families, called $\mathfrak{S}$-families, have the following properties:

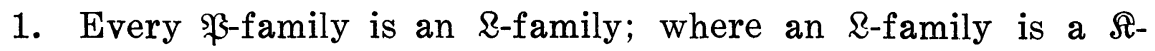
family satisfying the two conditions:

(a) every $\mathscr{C}$-set is a $\mathscr{C}_{I I}$-set,

(b) every countable set is a $\mathscr{C}_{I}$-set.

2. Every Borel set has the Baire property with respect to $(X, \mathscr{C})$.

Property 1 we have referred to in [14] as Baire's theorem, 
since it is a generalization of the so-called Baire Category Theorem according to which the real line cannot be represented as a countable union of nowhere dense sets. W. H. Young has pointed out that this latter theorem should be called the Baire-Osgood theorem, because it was actually discovered about the same time by W. F. Osgood, independently of R. Baire (cf. [15] p. 290, [16] p. 173, [1] p. 65, [17] p. 462, and [27] p. 426, 428). However, the method usually employed to prove this theorem, by successively avoiding a sequence of nowhere dense sets and thereby determining a descending sequence of closed intervals, goes back at least to 1870 (see [8] p. 90), and the so-called Baire Category Theorem might be more appropriately attributed to someone other than Baire and Osgood (see also [2] §50).

We now recall the three most important examples of $\$$-families.

ExAmple 1A. $\mathscr{C}$ is the family of all bounded, closed intervals $[a, b]$. As shown in [14], the absolute Baire property with respect to $(X, \mathscr{C})$ coincides with the topological Baire property in the restricted sense and the absolute $\mathscr{C}_{I}$-sets coincide with the sets always of the first category.

EXAMPLE 2A. $\mathscr{C}$ is the family of all perfect sets which are of positive Lebesgue measure in every neighborhood of each of their points. The absolute Baire property here is identical to the notion of absolute measurability and the absolute $\mathscr{C}_{I}$-sets are identical to the absolute null-sets.

EXAMPLE 3A. $\mathscr{C}$ is the family of all perfect sets. In this case, the sets which have the absolute Baire property are the same as the sets having the property $(s)$ of Marczewski and the absolute $\mathscr{C}_{1}$-sets are the same as the sets having Marczewski's property $\left(s^{\circ}\right)$ (see [24]).

In order to motivate the definition of the topological version of the absolute Baire property given in the next section, we shall show how one may obtain from any $\mathfrak{P}$-family $(X, \mathscr{C})$ of subsets of the real line $X$, a $\Omega$-family $(Z, \mathscr{Z})$ of subsets of the set $Z$ of all irrational numbers such that $\mathfrak{A}(\mathscr{C})=\mathfrak{A}(\mathscr{Z})$ and the absolute $\mathscr{C}_{I}$-sets coincide with the absolute $\mathscr{\varkappa}_{I}$-sets.

M. Fréchet ([7], Note I) has proved, using measure-theoretic methods, a theorem which has as an immediate consequence the fact that every linear perfect set can be represented as a disjoint union of a set of order type $\lambda$, the order type of the real line, and a countable set; e.g., upon removing from a given perfect set $P$ its first and last elements, if any, and all points which are only right-hand-side 
limit points of $P$, we obtain a set $\lambda(P)$ of order type $\lambda$. In [14] $\S 3$, another proof of this fact was given, devoid of measure-theoretic notions, and a certain subset $D$ of $\lambda(P)$ was shown to be order-dense in $\lambda(P)$. This set $D$ can contain only points which are either rational numbers in $P$ or are only left-hand-side limit points of $P$ and if we remove $D$ from the set $\lambda(P)$, we obtain a set $\lambda(P)-D$ which can be mapped onto the set $Z$ by an order preserving homeomorphism.

For each perfect set $P$, let $\zeta(P)$ be the set obtained by removing from $P$ all points which are only unilateral limit points of $P$ and all rational numbers belonging to $P$. The set $\zeta(P)$, which may also be formed by removing all rational numbers from $\lambda(P)-D$, is thus order isomorphic and homeomorphic to $Z$.

Lemma 1. If $A$ and $B$ are perfect sets then $A \subset B$ if and only if $\zeta(A) \subset \zeta(B)$.

Proof. Assume $\zeta(A) \subset \zeta(B)$. If $x \in A$ then $x$ is a condensation point of $\zeta(A)$ and also of $\zeta(B)$. Therefore $x$ belongs to the perfect set $B$.

Conversely, assume $A \subset B$ and suppose $x \notin \zeta(B)$. We shall show $x \notin \zeta(A)$. Now either $x \notin A$ or $x \in A-\zeta(B)$. If $x \notin A$ then obviously, $x \notin \zeta(A)$. Thus assume $x \in A-\zeta(B)$. Since $x \in B-\zeta(B)$, either $x$ is a rational number belonging to $B$ or $x$ is a unilateral limit point of $B$. If $x$ is a rational number then we immediately have $x \notin \zeta(A)$. On the other hand, if $x$ is a unilateral limit point of $B$ and $x \in A$, then $x$ is also a unilateral limit point of $A$ and consequently $x \notin \zeta(A)$.

Without loss of generality, we can assume $X$ is an element of $\mathscr{C}$. For it can be easily seen that if $(X, \mathscr{C})$ is a $\$$-family then $(X, \mathscr{C} \cup\{X\})$ is an equivalent $\mathfrak{P}$-family.

TheOREM 1. Let $(X, \mathscr{C})$ be a $\mathfrak{B}$-family with $X \in \mathscr{C}$ and let

$$
\mathscr{Z}=\{\zeta(A): A \in \mathscr{C}\} \text {. }
$$

Then the following facts are obtained.

(i) $(Z, \mathscr{Z})$ is an \&-family, which we shall call the \&-family associated with the $\mathfrak{\beta}$-family $(X, \mathscr{C})$.

(ii) $\mathscr{Z}_{I}=\left\{S \in \mathscr{C}_{I}: S \subset Z\right\}=\left\{T \cap Z: T \in \mathscr{C}_{I}\right\}$

$$
\mathfrak{B}(\mathscr{b})=\{S \in \mathfrak{B}(\mathscr{C}): S \subset Z\}=\{T \cap Z: T \in \mathfrak{B}(\mathscr{C})\} .
$$

(iii) $\mathfrak{A}(\mathscr{Z})=\mathfrak{A}(\mathscr{C})$ and the absolute $\mathscr{Z}_{I}$-sets coincide with the absolute $\mathscr{C}_{I}$-sets.

Proof. An argument similar to that used to prove Lemma 4 [14] shows $(Z, \mathscr{Z})$ is a $\Re$-family and 


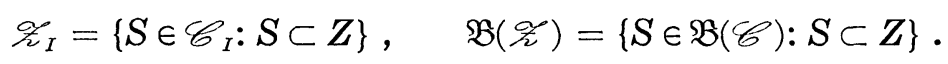

From these equalities it is easily seen that

$$
\mathscr{\mathscr { K }}_{I}=\left\{T \cap Z: T \in \mathscr{C}_{I}\right\}, \quad \mathfrak{B}(\mathscr{Z})=\{T \cap Z: T \in \mathfrak{B}(\mathscr{C})\},
$$

and $(Z, \mathscr{Z})$ is an \&-family.

Assume now that $S$ is a set which has the absolute Baire property with respect to $(X, \mathscr{C})$ and let $\psi$ be an order isomorphism mapping $Z$ onto a set $U \subset X$. We shall show $S \cap U \in \mathfrak{B}[\psi(\mathscr{Z})]$.

Applying Lemma 5 [14], extend $\psi$ to an order isomorphism $\phi$ mapping $X$ onto an ordinally closed set $V$ containing $U$.

Suppose $M=\psi(E)$, with $E \in \mathscr{Z}$, is a $\psi(\mathscr{Z})$-set and $S \cap U$ is a $\psi(\mathscr{Z})_{I I}$-set everywhere on $M$. The set $A=\bar{E}$, where $\bar{E}$ denote the closure of $E$, will then be a $\mathscr{C}$-set. We shall first show $S \cap U$ is a $\phi(\mathscr{C})_{I I}$-set everywhere on the $\phi(\mathscr{C})$-set $N=\dot{\phi}(A)$.

Let $P=\phi(B)$, with $B \in \mathscr{C}$, be any $\phi(\mathscr{C})$-set contained in $N$. Since $B \subset A$, the set $F=\zeta(B)$ is a $\mathscr{Z}$-set contained in $E=\zeta(A)$ and, setting $Q=\psi(F)$, we know $(S \cap U) \cap Q$ is a $\psi(\mathscr{Z})_{I I}$-set. From the easily established equality $\psi(\mathscr{\varkappa})_{I I}=\psi\left(\mathscr{Z}_{I I}\right)$ it follows that $(S \cap U) \cap$ $Q=\psi(T)$, where $T$ is a $\mathscr{L}_{I I}$-set and hence is also a $\mathscr{C}_{I I}$-set. Using the equality $\phi(\mathscr{C})_{I I}=\phi\left(\mathscr{C}_{I I}\right)$, we see that $(S \cap U) \cap Q=\phi(T)$ is a $\phi(\mathscr{C})_{I I}$-set. Thus, $(S \cap U) \cap P$ is a $\phi(\mathscr{C})_{I I}$-set for every $\phi(\mathscr{C})$-set $P \subset N$.

As $S \cap V \in \mathfrak{B}[\phi(\mathscr{C})]$ and $S \cap U$ is a $\phi(\mathscr{C})_{I I}$-set everywhere on $N$, there is a $\phi(\mathscr{C})$-set $P \subset N$ such that $P \cap[V-(S \cap U)]$ is a $\phi(\mathscr{C})_{I}$-set. Let $P=\phi(C)$ where $C$ is a $\mathscr{C}$-set. The set $L=\psi(\zeta(C))$ is a $\psi(\mathscr{Z})$-set contained in $M$ and $L \cap[U-(S \cap U)]$, which is contained in $P \cap$ $[V-(S \cap U)]$, is a $\psi(\mathscr{Z})_{T}$-set. Therefore $S \cap U \in \mathfrak{B}[\psi(\mathscr{Z})]$ and we conclude $S$ has the absolute Baire property with respect to $(Z, \mathscr{Z})$.

Conversely, assume $S$ is a set which has the absolute Baire property with respect to $(Z, \mathscr{Z})$ and let $\phi$ be any order isomorphism mapping $X$ onto a set $V \subset X$. We show $S \cap V \in \mathfrak{B}[\phi(\mathscr{C})]$.

Suppose $M=\phi(A)$, with $A \in \mathscr{C}$, is a $\phi(\mathscr{C})$-set and $S \cap V$ is a $\phi(\mathscr{C})_{I I}$-set everywhere on $M$. The restriction $\psi=\phi \mid Z$ of $\phi$ is an order isomorphism mapping $Z$ onto a set $U \subset V$ and $S \cap U$ is a $\psi(\mathscr{Z})_{I I}$-set everywhere on the $\psi(\mathscr{Z})$-set $N=\psi(\zeta(A)) \subset M$. Since $S \cap$ $U \in \mathfrak{B}[\psi(\mathscr{Z})]$, there is a $\psi(\mathscr{Z})$-set $P \subset N$ such that $P \cap(U-S)$ is a $\psi(\mathscr{C})_{I}$-set. Let $P=\psi(B)$, with $B \in \mathscr{Z}$. The set $L=\phi(\bar{B})$, where $\bar{B}$ denotes the closure of $B$, is a $\phi(\mathscr{C})$-set contained in $M$ and $L \cap$ $(V-S)$, which differs from $P \cap(U-S)$ by at most a countable set, is a $\dot{\phi}(\mathscr{C})_{I}$-set. Therefore $S \cap V \in \mathfrak{B}[\phi(\mathscr{C})]$ and we conclude $S$ has the absolute Baire property with respect to $(X, \mathscr{C})$.

Finally, it is easily seen from the equalities $\phi(\mathscr{C})_{I}=\phi\left(\mathscr{C}_{I}\right)$ and $\psi(\mathscr{\mathscr { L }})_{I}=\psi\left(\mathscr{\mathscr { C }}_{I}\right)$ that the absolute $\mathscr{\mathscr { Z }}_{I}$-sets coincide with the absolute 
$\mathscr{C}_{1}$-sets.

As a consequence of this theorem, we see that we can unify the concepts of the classical Baire property in the restricted sense, absolute measurability, and Marczewski's property (s) by taking $K=Z$ (and $\mathscr{C}=\mathscr{Z}$ ) in Definition 4 . We are thus led to introduce the following definition, which is a special case of Definition 4 to which we have added the notion of an absolute $\mathscr{C}$-singular set.

Definition 5. Let $(Z, \mathscr{C})$ be a $\Re$-family. A set $S \subset X$ has the absolute Baire property with respect to $(Z, \mathscr{C})$ [respectively, is an absolute $\mathscr{C}_{I}$-set, is an absolute $\mathscr{C}$-singular set] if for every order isomorphism $\phi$ mapping $Z$ into $X$, the set $S \cap \phi(Z)$ has the Baire property with respect to $(\phi(Z), \phi(\mathscr{C}))$ [respectively, is a $\dot{\phi}(\mathscr{C})_{I}$-set, is a $\dot{\phi}(\mathscr{C})$-singular set].

Denoting by $(Z, \mathscr{Z})$ the $\mathbb{R}$-family associated with the $\mathfrak{P}$-family $(X, \mathscr{C})$, we see that in Examples $2 \mathrm{~A}$ and $3 \mathrm{~A}$, the absolute $\mathscr{Z}$-singular sets are the same as the absolute $\mathscr{Z}_{I}$-sets. In Example $1 \mathrm{~A}$ however, these two classes of sets are distinct, with the class of absolute $\mathscr{\not}-$ singular sets coinciding with the class of ordinally scattered sets. The notion of an ordinally scattered set was introduced by $F$. Hausdorff (see [9] p. 458) who defined a set to be ordinally scattered, or in his terminology a 'zerstreute Menge', if it has no order-dense subset (with at least two elements). Alternatively, the ordinally scattered sets may be characterized as the sets which have no subset order isomorphic to the set of all rational numbers.

We shall refer to Definition 5 as the ordinal version of the absolute Baire property.

3. The absolute Baire property: topological version. Upon replacing in Definition 5 the words "order isomorphism" by the word "homeomorphism", we obtain the following definition which we call the topological version of the absolute Baire property.

Definition 6. Let $X$ be a topological space and let $(Z, \mathscr{C})$ be a $\Re$-family of subsets of the set $Z$ of all irrational numbers. A set $S \subset X$ has the absolute Baire property with respect to $(Z, \mathscr{C})$ [respectively, is an absolute $\mathscr{C}_{1}$-set, is an absolute $\mathscr{C}$-singular set] if for every homeomorphism $\phi$ mapping $Z$ into $X$, the set $S \cap \phi(Z)$ has the Baire property with respect to $(\phi(Z), \phi(\mathscr{C}))$ [respectively, is a $\phi(\mathscr{C})_{I^{-}}$ set, is a $\phi(\mathscr{C})$-singular set].

We now proceed to show that this definition embodies the classical Baire property in the restricted sense, absolute or universal measur- 
ability, and Marczewski's property (s), at least in the setting of a complete separable metric space $X$ which is what we assume $X$ to be for the remainder of this section.

ExAMPLe 1B. Let $(Z, \mathscr{Z})$ be the \&-family associated with the $\mathfrak{P}$-family of Example $1 \mathrm{~A}$.

Recall that a set $S \subset X$ has the classical Baire property relative to a set $E \subset X$ if every set $G$ open relative to $E$ contains a set $H$ open relative to $E$ such that either $H \cap S$ or $H \cap(E-S)$ is of the first category relative to $E$. For each set $E \subset X$, let $\mathscr{R}(E)$ denote the family of all subsets of $E$ which have the classical Baire property relative to $E$ and let $\mathscr{S}(E)$ denote the family of all subsets of $E$ which are of the first category relative to $E$.

Originally, the terminology "Baire property" was used to refer to what is presently called the "Baire property in the restricted sense" (see [21] p. 319 and [22] p. 38). A set $S \subset X$ has the Baire property in the restricted sense if $S \cap P$ has the Baire property relative to $P$, for every perfect set $P \subset X$. In the event that $S \cap P$ is of the first category relative to $P$, for every perfect set $P \subset X$, then, following Lusin ([11] p. 115), $S$ is said to be always of the first category.

Denjoy (cf. [4] p. $765 \mathrm{fn}$, [5] p. $156 \mathrm{fn}$, [6]) has called 'ensemble clairsemé' any set $S \subset X$ with the property that $S \cap P$ is nowhere dense relative to $P$, for every perfect set $P$. Such a set is called a scattered set, in English, and coincides with Cantor's earlier notion of a 'separierte Menge', which is a set which has no (nonempty) dense-in-itself subset (cf. [3] p. 228). Alternatively, the scattered sets may be characterized as the sets which have no subset homeomorphic to the set of all rational numbers. Further equivalent conditions characterizing the scattered sets may be found in [19].

A linear scattered set is not necessarily an ordinally scattered set. For example, the set of all midpoints of the intervals complementary to the Cantor set is a scattered set which is not an ordinally scattered set.

However, every linear set which is ordinally scattered must be a scattered set. For, by repeated application of the fact that if $x$ is any element of a set $T$ homeomorphic to the set of all rational numbers which is neither a first nor a last element of $T$, then each of the sets $\{t \in T: t\langle x\}$ and $\{t \in T: t>x\}$ is a denumerable dense-initself set and hence, according to a theorem of Sierpiński [20], is homeomorphic to the set of rational numbers, one can determine, in any set $S$ which is not scattered, a denumerable subset of points $x$ which is order isomorphic to the set of rational numbers, to conclude the set $S$ is not ordinally scattered. 
In the proof of our next theorem, we shall make use of the following elementary fact.

Lemma 2. If $P$ is a perfect set in $X$ and $E$ is a Borel subset of $P$ such that $P-E$ is of the first category relative to $P$, then for every set $S \subset X$

(i) $S \cap E \in \mathscr{S}(E)$ if and only if $S \cap P \in \mathscr{S}(P)$.

(ii) $S \cap E \in \mathscr{R}(E)$ if and only if $S \cap P \in \mathscr{R}(P)$.

THEOREM 2. If $X$ is a complete separable metric space then the sets which have the absolute Baire property with respect to $(Z, \mathscr{Z})$, the absolute $\mathscr{Z}_{I}$-sets, and the absolute $\mathscr{Z}$-singular sets coincide, respectively, with the subsets of $X$ which have the Baire property in the restricted sense, the sets always of the first category, and the scattered sets.

Proof. Let $P$ be a given perfect set in $X$. By a theorem of Marczewski ([26] Auxiliary Theorem I), there is a set $E$ which is a $\mathscr{G}_{j}$-set relative to $P$ such that $P-E$ is of the first category relative to $P$ and there is a homeomorphism $\phi$ mapping $Z$ onto $E$. If $S \subset X$ has the absolute Baire property with respect to $(Z, \mathscr{Z})$ then $S \cap E \in$ $\mathfrak{B}[\phi(\mathscr{Z})]=\mathscr{R}(E)$ and, by Lemma $2, S \cap P \in \mathscr{R}(P)$. If $S$ is an absolute $\mathscr{F}_{I}$-set then $S \cap E \in \phi(\mathscr{F})_{I}=\mathscr{S}(E)$ and hence $S \cap P \in \mathscr{P}(P)$. Therefore, if $S \subset X$ has the absolute Baire property with respect to $(Z, \mathscr{Z})$ then $S$ has the Baire property in the restricted sense and if $S$ is an absolute $\mathscr{Z}_{I}$-set then $S$ is always of the first category.

Conversely, let $\phi$ be a homeomorphism mapping $Z$ onto a set $E \subset X$. Each point of $E$ will be a condensation point of $E$ and consequently the closure of $E$ in $X$ is a perfect set $P$. Moreover, $E$ being a dense $\mathscr{G}_{j}$-set in $P$, the set $P-E$ is of the first category relative to $P$. Hence if $S \subset X$ has the Baire property in the restricted sense then $S \cap P \in \mathscr{R}(P)$ and, by Lemma $2, S \cap E \in \mathscr{R}(E)=\mathfrak{B}[\phi(\mathscr{Z})]$. If $S$ is always of the first category then $S \cap P \in \mathscr{S}(P)$ and consequently $S \cap E \in \mathscr{S}(E)=\phi(\mathscr{Z})_{I}$. Thus, if $S$ has the Baire property in the restricted sense then $S$ has the absolute Baire property with respect to $(Z, Z)$ and if $S$ is always of the first category then $S$ is an absolute $\mathscr{Z}_{I}$-set.

It now remains only to show that the absolute of-singular sets coincide with the scattered sets. In the proof of this fact we shall use the letters $Q$ and $R$ to denote the sets of rational and real numbers respectively.

Assuming $S$ is not scattered, there exists a homeomorphism $f$ mapping $Q$ onto a set $T \subset S$. Apply Lavrentiev's theorem to extend $f$ to a homeomorphism $f^{*}$ between $\mathscr{G}_{i}$-sets $Q^{*}$ and $T^{*}$, with $Q \subset$ 
$Q^{*} \subset R$ and $T \subset T^{*} \subset X$. It follows from a theorem of Mazurkiewicz (see [10] p. 441-442) that upon the removal from $Q^{*}$ of an at most countable set of irrational numbers we obtain a set $Q^{* *}$, with $Q \subset$ $Q^{* *} \subset Q^{*}$, which is homeomorphic to $Z$. Let $g$ be a homeomorphism mapping $Z$ onto $Q^{* *}$ and set $\phi=f^{*} \circ g$. Since $g^{-1}(Q)$ is dense in $Z$, the set $\phi\left(g^{-1}(Q)\right)=f^{*}(Q)=T$ is dense in $\dot{\phi}(Z)$ and consequently $S \cap \dot{\phi}(Z)$ is not $\phi(\mathscr{N})$-singular. Therefore the set $S$ is not an absolute $\mathscr{Z}$-singular set.

Conversely, suppose $S$ is not an absolute $\mathscr{X}$-singular set. Then there exists a homeomorphism $\phi$ mapping $Z$ into $X$ and a $\phi(\not{Z})$-set $E$ such that $S \cap F$ is nonempty for every $\phi(\mathscr{L})$-set $F \subset E$. Denoting by $\Sigma$ the set of all (nonempty) finite sequences of 0 's and 1 's, we construct inside $A=\phi^{-1}(E)$ a dyadic schema $\left\langle A_{\sigma}\right\rangle_{\sigma \in \Sigma}$ of $\mathscr{2}$-sets satisfying the conditions:

(a) if $\sigma, \tau \in \Sigma$ and $\sigma$ is an initial section of $\tau$, then $A_{\tau} \subset A_{\sigma}$.

(b) if $\sigma, \tau \in \Sigma, \sigma \neq \tau$, and both have the same length $A_{\sigma}$ and $A_{\tau}$ are disjoint.

(c) if $\sigma \in \Sigma$ and $\sigma$ has length $n$, then $\operatorname{diam}\left(A_{\sigma}\right) \leqq 1 / n \operatorname{diam}(A)$.

(d) if $\sigma \in \Sigma, \sigma$ has a length $n$, and $\Sigma(n+1, \sigma)$ is the set of all sequences of 0 's and 1's of length $n+1$ which have $\sigma$ as an initial section, then $A_{\sigma}=\bigcup\left\{A_{\tau}: \tau \in \Sigma(n+1, \sigma)\right\}$.

Let $T$ be a set formed by choosing one point in each of the sets $S \cap \phi\left(A_{\sigma}\right)$, for all $\sigma \in \Sigma$. The set $T$ is homeomorphic under $\dot{\phi}$ to a denumerable, dense-in-itself subset of $Z$ and hence is homeomorphic to $Q$. Therefore, $S$ is not scattered.

EXAMPLe 2B. Let $(Z, \mathscr{Z})$ be the \&-family associated with the $\mathfrak{P}$-family of Example 2A.

For each set $E \subset X$, let $\mathscr{B}(E)$ denote the family of all Borel subsets of $E$. If $E$ is a Borel set in $X$ and $\mu$ is a measure on $(E, \mathscr{B}(E))$ then we shall denote by $\mathscr{C}(\mu)$ the family of all subsets of $E$ which are measurable with respect to the completion $\bar{\mu}$ of $\mu$ and by $\mathscr{N}(\mu)$ the family of all subsets of $E$ which are of measure zero with respect to the completion $\bar{\mu}$ of $\mu$.

A set $S \subset X$ is called absolutely measurable if $S \in \mathscr{C}(\mu)$ for every continuous probability measure $\mu$ on $(X, \mathscr{B}(X))$ and is called an absolute null-set if $S \in \mathscr{N}(\mu)$ for every such measure $\mu$. In Euclidean spaces, the absolutely measurable sets (respectively, the absolute null-sets) coincide with those sets every homeomorphic image of which is Lebesgue measurable (respectively, of Lebesgue measure zero) (cf. [25], especially $\S 4.2$ (iii), and [12]).

The family $\mathfrak{B}(\mathscr{\mathscr { L }})$ of sets which have the Baire property with respect to $(Z, \mathscr{Z})$ is the family of Lebesgue measurable subsets of $Z$ 
and the $\mathscr{Z}_{I}$-sets are the subsets of $Z$ of Lebesgue measure zero. We shall denote by $\lambda$ a continuous probability measure defined on $(Z, \mathscr{B}(Z))$ such that $\mathscr{M}(\lambda)=\mathscr{B}(\mathscr{Z})$ and $\mathscr{N}(\lambda)=\mathscr{Z}_{I}$. The existence of such a measure $\lambda$ follows from the fact that there exists a Lebesgue measurability-preserving homeomorphism between $Z$ and the set of all irrational numbers in the unit interval.

THEOREM 3. If $X$ is a complete separable metric space then the subsets of $X$ which have the absolute Baire property with respect to $(Z, \mathscr{C})$ are the same as the absolutely measurable sets and the absolute $\mathscr{Z}_{1}$-sets, as well as the absolute $\mathscr{F}_{\text {- }}$-singular sets, are the same as the absolute null-sets.

Proof. Let $\mu$ be a continuous probability measure on $(X, \mathscr{B}(X))$. It is a consequence of Theorem 2 [18] of J. Oxtoby, that there exists a homeomorphism $\phi$ mapping $Z$ onto a $\mathscr{G}_{j}$-set $E \subset X$ such that $\mu(X-E)=0, \phi[\mathscr{L}(\lambda)]=\mathscr{C}\left(\mu_{E}\right)$, and $\phi[\mathscr{N}(\lambda)]=\mathscr{N}\left(\mu_{E}\right)$, where $\mu_{E}$ denotes the restriction of $\mu$ to $(E, \mathscr{B}(E))$. We then have

$$
\begin{aligned}
& \mathfrak{B}[\dot{\phi}(\mathscr{L})]=\phi[\mathfrak{B}(\mathscr{\mathscr { L }})]=\phi[\mathscr{C}(\lambda)]=\mathscr{H}\left(\mu_{E}\right)=\{S \cap E: S \in \mathscr{C}(\mu)\}, \\
& \phi(\mathscr{Z})_{I}=\phi(\mathscr{\mathscr { Z }})=\phi[\mathscr{N}(\lambda)]=\mathscr{N}\left(\mu_{E}\right)=\{S \cap E: S \in \mathscr{N}(\mu)\} .
\end{aligned}
$$

Therefore, if $S \subset X$ has the absolute Baire property with respect to $(Z, \mathscr{Z})$ then $S$ is absolutely measurable and if $S$ is an absolute $\mathscr{Z}_{I^{-}}$ set then $S$ is an absolute null-set.

Suppose now that $\phi$ is a homeomorphism mapping $Z$ onto a set $E \subset X$. Let $\mu=\lambda \phi^{-1}$ be the measure on $(E, \mathscr{B}(E))$ induced by $\phi$ and $\lambda$; i.e., $\mu(B)=\lambda\left[\phi^{-1}(B)\right]$ for each $B \in \mathscr{B}(E)$. The measure $\mu$ is a continuous probability measure on $\mathscr{B}(E)$ which is positive on sets open in $E$. Moreover, $\mathscr{M}(\mu)=\mathfrak{B}[\dot{\phi}(\mathscr{Z})]$ and $\mathscr{N}(\mu)=\phi(\mathscr{Z})_{I}$. Extend $\mu$ to a continuous probability measure $\hat{\mu}$ on $(X, \mathscr{B}(X))$ by defining $\hat{\mu}(B)=\mu(B \cap E)$ for each $B \in \mathscr{B}(X)$. Then $\mathscr{M}(\mu)=\{S \cap E: S \in \mathscr{M}(\hat{\mu})\}$ and $\mathscr{N}(\mu)=\{S \cap E: S \in \mathscr{N}(\hat{\mu})\}$. Therefore, if $S \subset X$ is absolutely measurable then $S$ has the absolute Baire property with respect to $(Z, \mathscr{Z})$ and if $S$ is an absolute null-set then $S$ is an absolute $\mathscr{Z}_{I}$-set.

ExAmple 3B. Let $(Z, \mathscr{Z})$ be the $\&$-family associated with the \$-family of Example $3 \mathrm{~A}$.

We call a set $S \subset X$ a Marczewski set if every perfect set $P \subset X$ contains a perfect set $Q$ such that either $Q \subset S$ or $Q \subset X-S$, and call $S$ a Marczewski singular set if every perfect set $P$ contains a perfect set $Q$ disjoint from $S$. These sets coincide with the sets having the properties $(s)$ and $\left(s^{\circ}\right)$, respectively, introduced by Marczewski in [24]. In view of the fact that every perfect set in 
$X$ contains a set homeomorphic to $Z$, and vice versa, we may replace the perfect sets in the foregoing definition by sets homeomorphic to $Z$, to obtain an equivalent definition which we shall use below.

THEOREM 4. If $X$ is a complete separable metric space then the sets which have the absolute Baire property with respect to $(Z, \mathcal{Z})$ coincide with the Marczewski sets and the absolute $\mathscr{Z}_{I}$-sets, which are the same as the absolute $\mathscr{Z}$-singular sets, coincide with the Marczewski singular sets.

Proof. Assume $S \subset X$ has the absolute Baire property with respect to $(Z, \mathscr{Z})$ and let $E \subset X$ be any set homeomorphic to $Z$; say $E=\phi(Z)$, where $\phi$ is a homeomorphism mapping $Z$ onto $E$. Since $S \cap E \in \mathfrak{B}[\phi(\mathscr{Z})]=\phi[\mathfrak{B}(\mathscr{Z})]$, the set $\phi^{-1}(S \cap E)$ belongs to the $\sigma$-field $\mathfrak{B}(\mathscr{Z})$ which consists of all linear Marczewski sets contained in $Z$. Hence there is a $\mathscr{Z}$-set $B \subset Z$ such that either $B \subset \phi^{-1}(S \cap E)$ or $B \subset Z-\phi^{-1}(S \cap E)$. It follows that $F=\phi(B)$ is a $\phi(\mathscr{Z})$-set contained in $E$ which is homeomorphic to $Z$, and either $F \subset S$ or $F \subset X-S$. Thus $S$ is a Marczewski set in $X$.

Conversely, suppose $S$ is a Marczewski set in $X$ and let $\phi$ be any homeomorphism mapping $Z$ onto a set $E \subset X$. Since the $\mathscr{G}_{j}$-set $E$ is a Marczewski set in $X$ and the intersection of two Marczewski sets is a Marczewski set, $S \cap E$ is a Marczewski set in $X$. If $A$ is any $\phi(\mathscr{2})$-set, then $A$ contains a set $C$ which is homeomorphic to $Z$ such that $C \subset S \cap E$ or $C \subset X-(S \cap E)$. Moreover, $C$ contains a $\phi(\mathscr{Z})$-set $B$. Now, from the inclusion $B \subset S \cap E$ we obtain $B \cap[X-(S \cap E)]=\varnothing$ and from the inclusion $B \subset X-(S \cap E)$ we obtain $B \cap(S \cap E)=\varnothing$. Hence $B$ is a $\phi(\mathscr{Z})$-set contained in $A$ and either $B \cap(S \cap E) \in \phi\left(\mathscr{Z}_{I}\right)=$ $\phi(\mathscr{Z})_{I}$ or $B \cap[X-(S \cap E)] \in \phi(\mathscr{Z})_{I}$; i.e., $S \cap E \in \mathfrak{B}[\phi(\mathscr{\digamma})]$.

Thus the sets which have the absolute Baire property with respect to $(Z, \mathscr{Z})$ coincide with the Marczewski sets. By a similar argument it is seen that the absolute $\mathscr{\varkappa}_{I}$-sets coincide with the Marczewski singular sets.

In [24] 5 , Marczewski has shown that, in a complete separable metric space, the $\sigma$-field of absolutely measurable sets and the $\sigma$-field of sets which have the classical Baire property in the restricted sense are contained in the $\sigma$-field of Marczewski sets. More generally, by an argument similar to that used to prove Theorem 21 [14], one can prove

THEOREM 5. Let $(Z, \mathscr{Z})$ be the \&-family associated with a $\$$ family of subsets of the real line. If $X$ is a complete separable metric space then the $\sigma$-field of subsets of $X$ which have the absolute Baire property with respect to $(Z, \mathscr{Z})$ is contained in the $\sigma$-field of 
Marczewski sets in $X$ and the $\sigma$-ideal of absolute $\mathscr{F}_{I}$-sets in $X$ is contained in the $\sigma$-ideal of Marczewski singular sets.

As we have seen above, Definitions 5 and 6 are not equivalent, since the absolute $\mathscr{C}$-singular sets of Examples $1 \mathrm{~A}$ and $1 \mathrm{~B}$ are not the same. There are, however, many theorems in the theory of linear ordered sets which have topological counterparts obtained by replacing ordinal concepts by corresponding topological concepts, and vice versa. Not only are the statements of these corresponding theorems analogous, but one can frequently give completely analogous proofs, in which any usage of Lavrentiev's theorem on extension of homeomorphisms is replaced by the usage of an analogous theorem on extension of order isomorphisms (see [14] Lemma 5). We shall not enter here into a detailed discussion of these analogies, but merely note that, with respect to this ordinal-topological duality, Definitions 5 and 6 are the appropriate analogues of one another.

3. Topological invariance. Theorem 27 of [14] established, under certain conditions, the invariance under order isomorphisms of the absolute Baire property as defined in $\S 2$. By a similar argument, we prove in this section a general theorem on the invariance under homeomorphisms of the absolute Baire property as defined in $\S 3$.

Definition 7. Let $(Z, \mathscr{C})$ be any $\Re$-family. A set $S \subset Z$ is called $\mathscr{C}$-homogeneous if for every point $x \in S$ and every open interval $I$ containing $x, S \cap I$ is a $\mathscr{C}_{I I}$-set.

We assume throughout this section that $(Z, \mathscr{C})$ is a $\Re$-family which is equivalent to a family $(Z, \mathscr{Z})$ that has been derived from a $\$$-family of perfect subsets of the real line in the manner given in Theorem 1. It then follows that all countable subsets of $Z$ are $\mathscr{C}_{I}$-sets, all Borel sets in $Z$ have the Baire property with respect to $(Z, \mathscr{C})$, and $Z$ is a $\mathscr{C}$-homogeneous set. Moreover, the sets which have the absolute Baire property with respect to $(Z, \mathscr{C})$ and the absolute $\mathscr{C}_{I}$-sets, in a given space $X$, are the same as the sets which have the absolute Baire property with respect to $(Z, \mathscr{Z})$ and the absolute $\mathscr{\varkappa}_{I}$-sets, respectively.

THEOREM 6. If $(Z, \mathscr{C})$ satisfies the conditions

(1) $\mathscr{C}$ is the set of all $\mathscr{C}$-homogeneous sets homeomorphic to $Z$,

(2) for every $\mathscr{C}$-set $A$, there is a homeomorphism \& mapping $Z$ onto $A$ such that $\psi(\mathscr{C})=\{B \in \mathscr{C}: B \subset A\}$, 
then the absolute Baire property with respect to $(Z, \mathscr{C})$ and the property of being an absolute $\mathscr{C}_{I}$-set are topologically invariant in complete metric spaces.

Proof. Assume $X$ is a complete metric space, $S$ is a subset of $X$, and $f$ is a homeomorphism mapping $S$ onto a set $T$ contained in a complete metric space $Y$. Apply Lavrentiev's theorem ([10] p. 429 ) to extend $f$ to a homeomorphism $g$ between $\mathscr{G}_{i}$-sets $S^{*}$ and $T^{*}$, where $S \subset S^{*} \subset X$ and $T \subset T^{*} \subset Y$.

Let $\phi$ be any homeomorphism mapping $Z$ onto a set $L=\phi(Z) \subset Y$ and suppose $E$ is a $\dot{\phi}(\mathscr{C})$-set on which $T \cap L$ is a $\phi(\mathscr{C})_{I I}$-set everywhere. We shall first establish the existence of a homeomorphism $h$ mapping $Z$ onto a set $K=h(Z) \subset X$ such that $K$ is an $h(\mathscr{C})$-set and $S \cap K$ is an $h(\mathscr{C})_{I I}$-set everywhere on $K$.

The set $E$, being homeomorphic to $Z$, is a $\mathscr{G}_{i}$-set, as is also the set $E \cap T^{*}$. According to a theorem of Mazurkiewicz (see [10] $\S 36$ ), upon the deletion of an at most countable set from $E \cap T^{*}$, we obtain a set $F$ which is homeomorphic to $Z$.

Now $F$ is a $\phi(\mathscr{C})_{I I}$-set everywhere on the $\dot{\phi}(\mathscr{C})$-set $E$. To see this, it suffices to show $E \cap T^{*}$ is a $\phi(\mathscr{C})_{I I}$-set everywhere on $E$, since $\left(E \cap T^{*}\right)-F$ is countable and consequently is a $\phi(\mathscr{C})_{I}$-set. If $C$ is any $\phi(\mathscr{C})$-set contained in $E$, then $(T \cap L) \cap C=T \cap C=C \cap$ $(E \cap T)$ is a $\phi(\mathscr{C})_{I I}$-set. Hence $C \cap\left(E \cap T^{*}\right)$ is a $\phi(\mathscr{C})_{I I}$-set for every $\dot{\phi}(\mathscr{C})$-set $C \subset E . \quad F$ being a $\phi(\mathscr{C})_{I I}$-set everywhere on the $\phi(\mathscr{C})$-set $E$, the set $A=\phi^{-1}(F)$ is a $\mathscr{C}_{I I}$-set everywhere on the $\mathscr{C}$ set $B=\phi^{-1}(E)$. We shall show $A$ is itself a $\mathscr{C}$-set.

Suppose $x \in A$ and $I$ is any open interval containing $x$, then $x$ belongs to the $\mathscr{C}$-set $B$ and $I \cap B$ is a $\mathscr{C}_{I I}$-set. By the Fundamental Theorem, $I \cap B$ is a $\mathscr{C}_{I I}$-set everywhere on some $\mathscr{C}$-set $C$. The set $B \cap C$ is then a $\mathscr{C}_{I I}$-set which, by Proposition 1 [14], contains a $\mathscr{C}$ set $D$. Since $I \cap B$ has the Baire property with respect to $\mathscr{C}$ and is a $\mathscr{C}_{I I^{-}}$-set everywhere on $D$, the set $D-(I \cap B)=D-I$ is a $\mathscr{C}_{I^{-}}$ set. It follows from the equality $A \cap D=[(A \cap D) \cap I] \cup[(A \cap D)-I]$ and the fact that $A$ is a $\mathscr{C}_{I I}$-set everywhere on $B$, hence also on $D$, that $(A \cap D) \cap I$ is a $\mathscr{C}_{I I}$-set. Therefore, $A \cap I$ is a $\mathscr{C}_{I I}$-set for every open interval $I$ containing $x$ and we conclude $A$ is a $\mathscr{C}$-set.

Let $\psi$ be a homeomorphism mapping $Z$ onto $A$ such that $\psi(\mathscr{C})=$ $\{B \in \mathscr{C}: B \subset A\}$ and set $h=g^{-1} \circ(\phi \circ \psi)$. It is easily seen that $\phi \circ \psi(\mathscr{C})=$ $\{G \in \phi(\mathscr{C}): G \subset F\}$ and, by Proposition 1 [14], that every $\dot{\phi} \psi(\mathscr{C})_{I^{-}}$ set $U \subset F$ is a $\phi(\mathscr{C})_{I}$-set. As $T \cap F$ is a $\phi(\mathscr{C})_{I I}$-set everywhere on $F$, the set $T \cap F$ is a $\phi \circ \psi(\mathscr{C})_{I I}$-set everywhere on $F$, and $g^{-1}(T \cap F)=$ $S \cap h(Z)$ is an $h(\mathscr{C})_{I I}$-set everywhere on $g^{-1}(F)=h(Z)$. Setting $K=h(Z)$, we have established the existence of the desired homeomorphism. 
It now follows immediately that if $S$ is an absolute $\mathscr{C}_{I}$-set, then $T=f(S)$ is also an absolute $\mathscr{C}_{I}$-set.

On the other hand, if $S$ has the absolute Baire property with respect to $(Z, \mathscr{C})$, there is an $h(\mathscr{C})$-set $H \subset g^{-1}(F)$ such that $H \cap$ $\left[g^{-1}(F)-g^{-1}(T \cap F)\right]$ is an $h(\mathscr{C})_{I}$-set; whence $D=g(H)$ is a $\phi \circ \psi(\mathscr{C})_{I^{-}}$ set. Therefore $D$ is a $\phi(\mathscr{C})$-set contained in $E$ and $D \cap[L-(T \cap L)]$ is a $\phi(\mathscr{C})_{I}$-set. The set $T \cap L=T \cap \phi(Z)$ is thus seen to have the Baire property with respect to $(\phi(Z), \phi(\mathscr{C}))$ for any homeomorphism $\phi$ mapping $Z$ into $Y$; hence $T=f(S)$ has the absolute Baire property with respect to $(Z, \mathscr{C})$.

EXAMPLE 1C. The topological invariance of the classical Baire property in the restricted sense was first established for Euclidean spaces by W. Sierpiński [21]. By means of Lavrentiev's theorem on the extension of homeomorphisms for complete metric spaces, Sierpiński's theorem readily extends to this more general situation (cf. [10] p. 432).

If we take $\mathscr{C}$ to be the family of all $\mathscr{G}_{\delta}$-sets in $Z$ which are homeomorphic to $Z$ and of the second category in $Z$ at each of their points, then we obtain a $\Re$-family $(Z, \mathscr{C})$ which is equivalent to the \&-family $(Z, \mathscr{Z})$ of Example $1 \mathrm{~B}$ and which satisfies conditions (1) and (2) of Theorem 6. In view of Theorem 2, we may then conclude from Theorem 6 .

COROLlaRY 1. The classical Baire property in the restricted sense and the property of a set being always of the first category are topologically invariant in complete separable metric spaces.

EXAMPLE 2C. The topological invariance of absolute measurability was established for complete separable metric spaces by W. Sierpiński and E. Szpilrajn/Marczewski (see [23], [25]).

Taking $\mathscr{C}$ to be the family of all $\mathscr{G}_{i}$-sets contained in $Z$ which are homeomorphic to $Z$ and of positive Lebesgue measure at each of their points, we obtain a $\Re$-family equivalent to the \&-family $(Z, \mathscr{Z})$ of Example 2B. Applying Theorems 3 and 6 , we obtain

COROLlaRY 2. Absolute measurability and the property of being an absolute null-set are topologically invariant in complete separable metric spaces.

EXAMPLE 3C. If $\mathscr{C}$ is the family of all $\mathscr{G}_{i}$-sets contained in $Z$ which are homeomorphic to $Z$, then $(Z, \mathscr{C})$ is a $\Re$-family equivalent to the \&-family $(Z, \mathscr{Z})$ of Example 3B. By means of Theorems 4 and 6 , we obtain the following theorem of E. Marczewski [24]. 
COROLLARY 3. The class of Marczewski sets and the class of Marczewski singular sets are topologically invariant in complete separable metric spaces.

\section{REFERENCES}

1. R. Baire, Sur les fonctions de variables réelles, Annali di Matematica, Serie 3, 3 (1899), 1-123.

2. P. du Bois-Reymond, Die allgemeine Functionentheorie, H. Laupp, Tübingen 1882; translated by G. Milhaud and A. Girot as Théorie générale des fonctions, Imprimerie Niçoise, Nice 1887.

3. G. Cantor, Gesammelte Abhandlungen, Georg Olms Verlagsbuchhandlung, Hildesheim 1966.

4. A. Denjoy, Sur les nombres dérivés, Comptes Rendus Hebdomadaires des Séances de l'Académie des Sciences, Paris, 160 (1915), 763-766.

5. - Mémoire sur les nombres dérivés des fonctions continues, Journal de Mathématiques Pures et Appliquées, Série 7, 1 (1915), 105-240.

6. - Sur les ensembles clairsemés, Koninklijke Akademie van Wetenschappen te Amsterdam, Proceedings of the Section of Sciences (Proceedings of the Royal Academy, Amsterdam) 22 (1920), 882-890; also appears in Verslagen van de Gewone Vergaderingen der Wis- en Natuurkundige, Afdeeling..., 28 (1920), 1100-1108.

7. M. Fréchet, Sur quelques points du calcul fonctionnel, Rendiconti del Circolo Matematico di Palermo, 22 (1906), 1-74.

8. H. Hankel, Untersuchungen über die unendlich oft oscillirenden und unstetigen Functionen, (Abdruck aus dem Gratulationsprogramm der Tübinger Universität vom 6 März 1870), Mathematische Annalen, 20 (1882), 63-112.

9. F. Hausdorff, Grundzüge einer Theorie der geordneten Mengen, Mathematische Annalen, 65 (1908), 435-505.

10. K. Kuratowski, Topology, Volume 1, Academic Press, New York, 1966.

11. N. Lusin, Sur les ensembles toujours de première catégorie, Fundamenta Mathematicae, 21 (1933), 114-126.

12. E. Marczewski, Remarque sur la mesurabilité absolue, Colloquium Mathematicum, $1(1947-8), 42-43$.

13. J. C. Morgan, II, Baire category from an abstract viewpoint, Fundamenta Mathematicae, 94 (1977), 13-23.

14. The absolute Baire property, Pacific J. Math., 65 (1976), 421-436.

15. W. F. Osgood, Ueber die ungleichmäßige Convergenz und die gliedwise Integration der Reihen, Nachrichten von der Königliche Gesellschaft der Wissenschaften zu Göttingen, Mathematisch-physikalische Klasse 1896, 288-291.

16. - Non-uniform convergence and the integration of series term by term, Amer. J. Math., 19 (1897), 155-190.

17. — Zweite Note über analytische Functionen mehrerer Veränderlichen, Mathematische Annalen, 53 (1900), 461-464.

18. J. C. Oxtoby, Homeomorphic measures in metric spaces, Proc. Amer. Math. Soc., 24 (1970), 419-423.

19. Z. Semadeni, Sur les ensembles clairsemés, Rozprawy Matematyczne, 19 (1959), 39 .

20. W. F. Sierpiński, Sur une propriété topologique des ensembles dénombrables denses en soi, Fundamenta Mathematicae, 1 (1920), 11-16.

21. - Sur l'invariance topologique de la propriété de Baire, ibid., 4 (1923), 319-323.

22. - Hypothèse du Continu, 2nd edition, Chelsea Publishing Company, New York 1956. 
23. W. Sierpiński and E. Szpilrajn, Remarque sur le problème de la mesure, Fundamenta Mathematicae, 26 (1936), 256-261.

24. E. Szpilrajn (Marczewski), Sur une classe de fonctions de M. Sierpinski et le classe correspondante d'ensembles, ibid., 24 (1935), 17-34.

25. - $O$ zbiorach $i$ funcjach bezwzglednie mierzalnych (On absolutely measurable sets and functions), Sprawozdania z Posiedzeń Towarzystwa Naukowego Warszawskiego, Wydział III (Comptes Rendus des Séances de la Société des Sciences et des Lettres de Varsovie, Classe III), 30 (1937), 39-68.

26. On the equivalence of some classes of sets, Fundamenta Mathematicae, 30 (1938), 235-241.

27. W. H. Young, The progress of mathematical analysis in the twentieth century, Proc. London Math. Soc., Series 2, 24 (1926), 421-434.

Received October 5, 1977 and in revised form March 23, 1978.

California State Polytechnic University

POMONA, CA 91768 



\title{
PACIFIC JOURNAL OF MATHEMATICS
}

\section{EDITORS}

RICHARD ARENS (Managing Editor)

University of California

Los Angeles, CA 90024

Charles W. Curtis

University of Oregon

Eugene, OR 97403

C. C. MOORE

University of California

Berkeley, CA 94720

\section{J. DUGUNDJI}

Department of Mathematics University of Southern California Los Angeles, CA 90007

R. Finn and J. Milgram

Stanford University

Stanford, CA 94305

\section{ASSOCIATE EDITORS}
E. F. BECKENBACH
B. H. NeumanN
F. WOLF
K. YOSHIDA

\section{SUPPORTING INSTITUTIONS}

\author{
UNIVERSITY OF BRITISH COLUMBIA \\ CALIFORNIA INSTITUTE OF TECHNOLOGY \\ UNIVERSITY OF CALIFORNIA \\ MONTANA STATE UNIVERSITY \\ UNIVERSITY OF NEVADA, RENO \\ NEW MEXICO STATE UNIVERSITY \\ OREGON STATE UNIVERSITY \\ UNIVERSITY OF OREGON
}

\author{
UNIVERSITY OF SOUTHERN CALIFORNIA \\ STANFORD UNIVERSITY \\ UNIVERSITY OF HAWAII \\ UNIVERSITY OF TOKYO \\ UNIVERSITY OF UTAH \\ WASHINGTON STATE UNIVERSITY \\ UNIVERSITY OF WASHINGTON
}

The Supporting Institutions listed above contribute to the cost of publication of this Journal, but they are not owners or publishers and have no responsibility for its content or policies.

Mathematical papers intended for publication in the Pacific Journal of Mathematics should be in typed form or offset-reproduced, (not dittoed), double spaced with large margins. Please do not use built up fractions in the text of the manuscript. However, you may use them in the displayed equations. Underline Greek letters in red, German in green, and script in blue. The first paragraph or two must be capable of being used separately as a synopsis of the entire paper. Items of the bibliography should not be cited there unless absolutely necessary, in which case they must be identified by author and journal, rather than by item number. Manuscripts, in triplicate, may be sent to any one of the editors. Please classify according to the scheme of Math. Reviews, Index to Vol. 39. All other communications should be addressed to the managing editor, or Elaine Barth, University of California, Los Angeles, California, 90024.

50 reprints to each author are provided free for each article, only if page charges have been substantially paid. Additional copies may be obtained at cost in multiples of 50 .

The Pacific Journal of Mathematics is issued monthly as of January 1966. Regular subscription rate: $\$ 72.00$ a year (6 Vols., 12 issues). Special rate: $\$ 36.00$ a year to individual members of supporting institutions.

Subscriptions, orders for numbers issued in the last three calendar years, and changes of address should be sent to Pacific Journal of Mathematics, P.O. Box 969, Carmel Valley, CA 93924, U.S.A. Older back numbers obtainable from Kraus Periodicals Co., Route 100, Millwood, NY 10546.

PUBLISHED BY PACIFIC JOURNAL OF MATHEMATICS, A NON-PROFIT CORPORATION

Printed at Kokusai Bunken Insatsusha (International Academic Printing Co., Ltd.). 8-8, 3-chome, Takadanobaba, Shinjuku-ku, Tokyo 160, Japan.

Copyright (C) 1978 by Pacific Journal of Mathematics Manufactured and first issued in Japan 


\section{Pacific Journal of Mathematics \\ Vol. 78, No. $2 \quad$ April, 1978}

Su-Shing Chen, Weak rigidity of compact negatively curved manifolds .... 273

Heinz Otto Cordes and D. A. Williams, An algebra of pseudodifferential operators with nonsmooth symbol ....................... 279

Herbert Paul Halpern, Normal expectations and integral decomposition of type III von Neumann algebras ......................... 291

G. Hochschild, On representing analytic groups with their

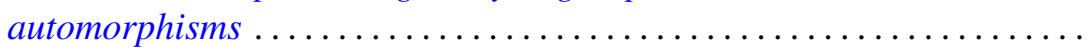

Dean G. Hoffman and David Anthony Klarner, Sets of integers closed under affine operators - the closure of finite sets ....................

Simeon Ivanov, On holomorphic relative inverses of operator-valued

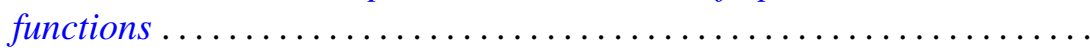

O. P. Juneja and M. L. Mogra, Radii of convexity for certain classes of

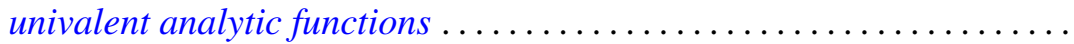

Hadi Kharaghani, The evolution of bounded linear functionals with application to invariant means.......................... 369

Jack W. Macki, A singular nonlinear boundary value problem .......... 375

A. W. Mason and Walter Wilson Stothers, Remarks on a theorem of $L$. Greenberg on the modular group ........................ 385

Kevin Mor McCrimmon, Peirce ideals in Jordan algebras . . . . . . . . . . . 397

John C. Morgan, II, On the absolute Baire property ................ 415

Gerard J. Murphy, Commutative non-Archimedean $C^{*}$-algebras ...

Masafumi Okumura, Submanifolds with L-flat normal connection of the

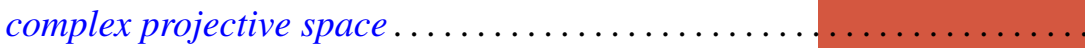

Chull Park and David Lee Skoug, Distribution estimates of barrier-crossing probabilities of the Yeh-Wiener process ...............

Irving Reiner, Invariants of integral representations ........

Phillip Schultz, The typeset and cotypeset of a rank 2 abelian group ..... 503

John Brendan Sullivan, Representations of Witt groups ....

Chia-Chi Tung, Equidistribution theory in higher dimensions . . . 